\title{
Morphological and immunohistochemical analysis of the human liver in chronic pancreatitis
}

\author{
R P Jalleh, J A Gilbertson, R C N Williamson, C S Foster
}

\begin{abstract}
Morphological and immunohistological appearances of liver biopsy specimens are described in a personal series of 52 patients undergoing operation for chronic pancreatitis. The findings are compared with those in a series of 10 histologically normal liver biopsy specimens from patients without pancreatitis. Alcohol was the prime aetiological agent in $\mathbf{4 0}$ of the 52 patients $(77 \%)$. No obvious damage to hepatic parenchymal cells or biliary structures was observed but minor morphological changes of alcohol associated liver disease were seen in $42 \%$ of specimens. The most consistent finding, present in 48 specimens $(92 \%)$, was a chronic inflammatory cell infiltration of portal tracts. In all but one case, $T$ lymphocytes predominated, but a few $B$ cells were present. In four biopsy specimens, $T$ cells spilled over into adjacent hepatic parenchyma, but there was no evidence of $T$ cell mediated cytotoxic damage to the parenchymal cells or biliary epithelium. It is suggested that these inflammatory cells are in transit from the pancreas through the liver via the portal circulation and may reflect the underlying pathogenesis of chronic pancreatitis rather than alcoholic liver disease.
\end{abstract}

Chronic pancreatitis affects approximately three per 100000 of the British population, although the incidence may be increasing along with the national consumption of alcohol. ${ }^{12}$ The continuing inflammatory process in the pancreas is characterised by irreversible morphological change, especially fibrosis and loss of acini. Alcohol is the dominant aetiological factor in chronic pancreatitis and contributes to several forms of liver disease, notably alcoholic hepatitis, cirrhosis, and hepatocellular carcinoma. Yet clinical evidence of a definite association between chronic pancreatitis and alcoholic liver disease is lacking. ${ }^{3-5}$ Indeed, in our own experience of about 100 alcoholic patients undergoing operation for severe chronic pancreatitis, the liver has nearly always looked normal at laparotomy.

The observed histopathological appearances of the liver in chronic pancreatitis include minimal non-specific changes in architecture and are not characteristic of alcohol related liver disease. ${ }^{36}$ Some of these changes have been attributed to extrahepatic biliary obstruction, which is present in up to $27 \%$ of patients with chronic pancreatitis. ${ }^{7-9}$ Therefore, we have employed conventional histological techniques, immunohistochemistry, and lymphocyte morphometry to characterise liver disease in a series of surgical patients with chronic pan- creatitis of both alcoholic and non-alcoholic aetiology.

This study aimed to determine whether the livers of patients with chronic pancreatitis exhibited morphological changes of ethanol toxicity comparable with the severity of their pancreatic disease. If not, the objective was to identify morphological features, consistently expressed, that might indicate alternative pathogenetic mechanisms in chronic pancreatitis.

\section{Methods}

\section{PATIENTS}

Liver biopsy specimens were taken from an unselected personal series of 52 patients undergoing operation for chronic pancreatitis between December 1985 and June 1990 at Bristol Royal Infirmary or the Hammersmith Hospital, London. There were 40 men and 12 women with a mean age of 41.2 years. Detailed social histories indicated that alcohol was the cause in 40 patients, with mean (SD) estimated daily ethanol consumption of 216 (32) g over a period of $12 \cdot 8$ $(1 \cdot 4)$ years. Causes in the other 12 patients included previous acute pancreatitis in five and choledochal cyst in one; no cause was identified in the remaining six patients. Liver function tests were abnormal in only seven patients, and none were jaundiced at the time of operation. Operations were performed by a single surgeon (RCNW) and comprised pancreatic resection (44), pancreaticojejunostomy (5), and sphincteroplasty (3). Liver biopsy specimens were obtained intraoperatively using a Tru-cut biopsy needle (Travenol Laboratories, Illinois, USA), and more than one specimen was obtained in most cases.

\section{CONTROL LIVER SPECIMENS}

Ten histologically normal liver biopsy specimens taken from patients without pancreatitis were used as control tissues. Seven specimens were from patients with no known focal or generalised disease who were undergoing surgery after trauma. Two specimens were taken during distal pancreatectomy: one for a solitary neuroendocrine tumour and the other for removal of a benign pseudocyst. One specimen was taken from the unaffected lobe of liver during excision of a benign simple hepatic cyst.

\section{HISTOLOGICAL EXAMINATION}

Specimens were fixed in buffered neutral formol saline and embedded in paraffin wax. Tissue sections $(2 \mu \mathrm{m})$ were routinely stained with haematoxylin and eosin (H\&E) to identify 
TABLE I Histological and histochemical features of liver biopsy specimens

\begin{tabular}{lllcc}
\hline & \multicolumn{2}{l}{$\begin{array}{l}\text { Controls } \\
(n=10)\end{array}$} & \multicolumn{2}{l}{$\begin{array}{l}\text { Pancreatitis } \\
(n=52)\end{array}$} \\
\cline { 2 - 3 } \cline { 5 - 5 } Feature & $N o$ & $(\%)$ & $N o$ & $(\%)$ \\
\hline Parenchymal hyperplasia & 0 & - & 7 & $(13)$ \\
Mallory's hyaline & 0 & - & 0 & - \\
Steatosis & 0 & - & 18 & $(35)$ \\
Pericentral fibrosis & 0 & - & 4 & $(8)$ \\
Cholestasis & 0 & - & 5 & $(10)$ \\
PAS & 0 & - & 0 & - \\
Iron & 0 & - & 11 & $(21)$ \\
Copper & 0 & - & 2 & $(4)$ \\
Copper-associated protein & 0 & - & 0 & - \\
Portal inflammation & 3 & $(30)$ & 48 & $(92)$ \\
Marginal ductular proliferation & 0 & - & 4 & $(8)$ \\
Portal fibrosis & 0 & - & 0 & - \\
Cirrhosis & 0 & - & 0 & - \\
\hline
\end{tabular}

PAS = periodic acid sehiff

morphological features recognised to be associated with ethanol toxicity (Table I). Wherever feasible, these features were assessed using numerical grading systems which ranged from 0 -III according to whether none, $<50 \%$, $50-75 \%$, or $>75 \%$ respectively of hepatic parenchyma was affected. Parenchymal cell hyperplasia was defined as hepatocyte plates two or more cells thick and present in more than $15 \%$ of each specimen. Additional tissue sections were stained histochemically. Reticulin was identified by a modified Gordon and Sweet's technique. Periodic acid Schiff (PAS) staining before and after diastase digestion detected glycogen or intracellular glycoproteins including $\alpha_{1}$ antitrypsin. Perls' Prussian blue reaction was used to identify iron, which was quantified according to the criteria of Braillon et al. ${ }^{10}$ Copper and copper associated protein were identified by rhodanine and orcein respectively.

\section{IMMUNOHISTOCHEMISTRY}

Monoclonal antibody UCHLI (anti-human T cell) was obtained as a gift from Dr Peter Beverley, University College Hospital, London. Monoclonal antibody L26 (anti-human B cell) was purchased from Dako Ltd (High Wycombe, UK). UCHL1 was diluted 1:10 and L26 1:100 in $120 \mathrm{mmol} / \mathrm{l}$ sodium phosphate buffered saline (PBS, pH 7.4). Polyclonal rabbit antisera to lysozyme and to $\alpha_{1}$ antitrypsin (to identify macrophages) were purchased from Dako Ltd. Both were diluted to $1: 1000$ in PBS. A conventional indirect immunoperoxidase staining technique was used to detect bound monoclonal antibodies. Human tonsillar tissue was used as the positive control. Polyclonal antisera were detected by the peroxidase antiperoxidase technique with human splenic tissue as the positive control. These latter tissue sections were digested with trypsin $(0 \cdot 1 \%$ $\mathrm{w} / \mathrm{v}$ in aqueous $0.1 \% \mathrm{CaCl}_{2}$ for seven minutes at $37^{\circ} \mathrm{C}$ ) after dewaxing. Endogenous peroxidase was blocked with $0 \cdot 3 \% \mathrm{H}_{2} \mathrm{O}_{2}$ in distilled water for 30 minutes at room temperature. In all cases, sections were incubated with primary antibodies overnight at $4^{\circ} \mathrm{C}$. The resultant immune peroxidase complexes were developed in $0.05 \%$ 3,3'-diaminobenizidine hydrochloride (DAB, Aldrich, Gillingham, Dorset, UK) in PBS containing $0.03 \%$ (w/v) $\mathrm{H}_{2} \mathrm{O}_{2}$. Sections were counterstained with haematoxylin and mounted in Pertex (Histolab, Hemel Hempstead, UK).
TABLE II Immunohistochemical staining of mononuclear cells

\begin{tabular}{|c|c|c|c|c|c|}
\hline \multirow[b]{2}{*}{ Antibody } & \multicolumn{2}{|c|}{$\begin{array}{l}\text { Controls } \\
(n=10)\end{array}$} & \multicolumn{2}{|c|}{$\begin{array}{l}\text { Pancreatitis } \\
(n=52)\end{array}$} & \multirow[b]{2}{*}{ pvalue } \\
\hline & $N o$ & $(\%)$ & No & $(\%)$ & \\
\hline \multirow{4}{*}{$\begin{array}{l}\text { UCHL1 } \\
\text { (T lymphocytes) } \\
\text { L26 } \\
\text { (B lymphocytes) } \\
\text { Lysozyme } \\
\text { (macrophages) } \\
\alpha_{1} \text { Antitrypsin } \\
\text { (macrophages) }\end{array}$} & 3 & 30 & 47 & 90 & $\mathrm{p}<0.001$ \\
\hline & 0 & 0 & 8 & 15 & - \\
\hline & 10 & 100 & 43 & 83 & $p>0.5$ \\
\hline & 7 & 70 & 34 & 65 & $p>0.5$ \\
\hline
\end{tabular}

\section{MORPHOMETRY}

$B$ and $T$ lymphocytes in portal areas were counted using a $10 \times 10 \mathrm{~mm}$ graticule within the microscope ocular and a $\times 400$ magnification objective. For each specimen, the number of lymphocytes in three representative portal areas were counted and a mean value was calculated.

\section{STATISTICS}

$\chi^{2}$ and unpaired Student's $t$ test were used for statistical analysis of data.

\section{Results}

\section{MORPHOLOGY AND HISTOCHEMISTRY}

The morphological and histochemical appearances of 52 liver biopsy specimens from patients undergoing operation for chronic pancreatitis and from the 10 control liver biopsy specimens are shown in Table I. Immunohistochemical staining patterns of T cells, B cells, and macrophages are presented in Table II.

In all liver specimens examined, the hepatic architecture was intact and normal vascular relations were preserved. Parenchymal cell hyperplasia was not identified in any of the control specimens but was present in seven of the specimens (13\%) from chronic pancreatitis patients. Mallory hyaline bodies were not observed in any specimen.

In all biopsy specimens, the three portal tract elements were intact. No inflammation of bile ducts was seen and no vasculitis was identified. There was no appreciable increase in fibrous connective tissue, and cirrhosis was not present in any of the specimens. The most striking feature was the relative absence of inflammation from portal tracts of the control specimens whereas 47 of the $52(90 \%)$ specimens from patients with chronic pancreatitis contained portal tracts that had been infiltrated by increased numbers of mononuclear cells. The nature of these cellular infiltrates is detailed below.

\section{STEATOSIS}

Macrovesicular steatosis of the type associated with alcohol induced liver disease was not observed in any of the controls but was present in $18(35 \%)$ of biopsy specimens from patients with chronic pancreatitis. In 11 of these specimens, steatotic hepatocytes occupied less than $50 \%$ of the lobules (grade I). In the remaining seven specimens, fatty change occupied between 50 and $75 \%$ of the hepatic lobules (grade II). In 15 of 
the $18(83 \%)$ specimens showing fatty change, hepatic parenchyma and portal tracts contained less than 10 polymorph neutrophils per high power field.

PERICENTRAL FIBROSIS

A mild increase in pericentral venous fibrosis was present around some, but not all, central veins of the contained lobules in four $(8 \%)$ liver biopsy specimens from patients with chronic pancreatitis. No consistent association was found between perivenous fibrosis and any other of the commonly accepted characteristics of alcoholic liver disease.

\section{CHOLESTASIS}

Cholestasis was present in only five of the 52 liver biopsy specimens. In all of these, the distribution of bile was periportal and intracanalicular. No polymorph neutrophils were present, the appearances being those of non-inflammatory cholestasis. In only one of these five specimens was marginal ductular proliferation seen. No other morphological features of chronic biliary obstruction were identified in any of the specimens. Of the five patients in whom morphological features of cholestasis were identified, none were jaundiced and only two had twofold increases in serum bilirubin and alkaline phosphatase. However, all five had radiological evidence (endoscopic retrograde cholangiopancreatograph (4) hepatic iminodiacetic acid (HIDA) scan (1)) of extrahepatic bile duct obstruction.

\section{PAS STAINING}

Histochemical staining with PAS did not show intracellular PAS positive, diastase resistant globules within hepatocytes of any of the liver specimens examined. Nevertheless, in one biopsy specimen, prominent staining for $\alpha_{1}$ antitrypsin involving the hepatocytes indicated a probable heterozygous $\alpha_{1}$ antitrypsin deficiency (an incidence of $2 \%$ in this series).

\section{SIDEROSIS}

Hepatocellular siderosis was noted in 11 patients $(21 \%)$ and all were grade I. In three of these patients, Kupffer cells also contained stainable iron. Only two cases of siderosis were associated with steatosis, and in both the steatosis was mild.

Figure 1: Portal region of liver biopsy specimen stained using monoclonal antibody UCHL1. The portal tract is densely infiltrated with lymphocytes of $T$ cell lineage. There is spillage of Tlymphocytes into the adjacent parenchyma without evidence of cytolytic activity to portal structures or to adjacent hepatocytes (original magnification $\times 480)$.

\section{COPPER AND COPPER ASSOCIATED PROTEIN}

Intracellular copper was not found in any of the control biopsy specimens and was identified in only two specimens from pancreatitis patients using a rhodanine technique. In both specimens, staining was scanty (grade I). The appearances were not those of a congenital copper storage disease. Distribution was confined to a few periportal hepatocytes. No correlation was apparent between this staining and the presence of cholestasis, steatosis, or iron deposition. Orcein staining did not show stainable copper associated protein in any of the control or pancreatitis specimens.

\section{PORTAL INFLAMMATION}

In none of the 10 control liver biopsy specimens were acute inflammatory cells or eosinophils identified. In three of these, chronic inflammatory cells were seen. The phenotypes of these cells were determined by immunohistochemistry. However, 48 of the 52 specimens $(92 \%)$ from patients with chronic pancreatitis contained increased numbers of inflammatory cells. Neutrophils were present in only 15 specimens. Several eosinophils were identified within the portal tracts of one specimen in which cholestasis was also present. Neither neutrophils nor eosinophils made more than a scanty contribution to the inflammatory infiltrate within their respective specimens.

\section{MARGINAL DUCTULAR PROLIFERATION}

Marginal ductular proliferation was not present in any of the control biopsy specimens but was identified in four specimens from the patients with pancreatitis. In one of these specimens, marginal ductular proliferation was associated with cholestasis, while in the remaining three no cholestasis was evident.

PORTAL FIBROSIS

No increased portal fibrosis or cirrhosis were identified in any specimens from controls or pancreatitis patients.

\section{IMMUNOHISTOCHEMISTRY}

Immunohistochemistry was performed to identify and phenotype the mononuclear inflammatory cells in the liver biopsy specimens. Using the monoclonal antibody UCHL1, T lymphocytes were identified in only three of 10 control specimens $(30 \%)$ with a mean (SEM) density of 24 (4) cells per portal tract. T lymphocytes, however, were identified in 47 of $52(90 \%)$ specimens from pancreatitis patients. These cells were almost entirely located in the portal areas (Fig 1). The mean (SEM) number of T lymphocytes per portal tract was 45 (5) cells. In four specimens, isolated $\mathrm{T}$ lymphocytes were seen in sinusoidal and canalicular regions (Fig 2). In each of these specimens, there was an especially dense portal infiltration of T cells: mean (SEM) 130 (17) cells per portal tract. The relative density of $\mathrm{T}$ cell infiltration is shown in Table III. 
Figure 2: Focal collections of $T$ lymphocytes within hepatic parenchyma and without evidence of cytolytic activity to adjacent hepatocytes. No further organisation of these small cellular aggregates was observed and no granulomas were identified (original magnification $\times 480)$.

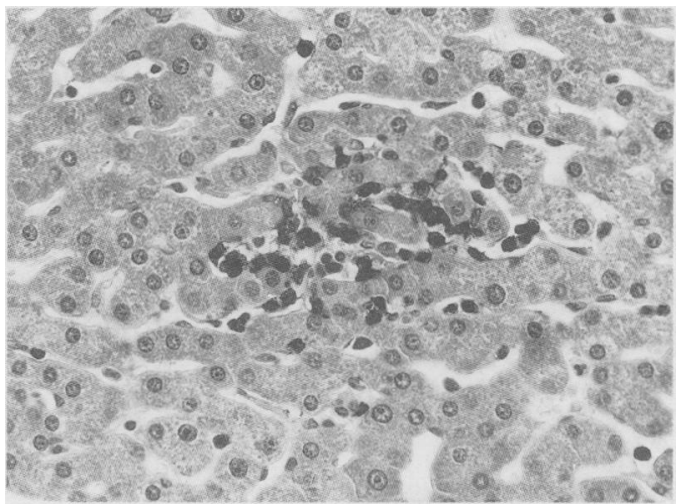

B lymphocytes were not identified in any of the control biopsy specimens. In contrast to the prevalence of $T$ cells in the liver biopsy specimens from patients with chronic pancreatitis, however, B lymphocytes were identified in only eight specimens $(15 \%)$. The pattern of infiltration was similar to that of $\mathrm{T}$ cells in that these lymphocytes were aggregated in portal regions. The density of this lymphoid infiltration was substantially less than that for $T$ cells, with a mean (SEM) of 10 (1) cells per portal area $(\mathrm{p}<0.001)$.

Macrophages were identified using polyclonal antisera (Table II). Positive staining for lysozyme was obtained in all control specimens and in 43 of the pancreatitis specimens $(83 \%)$.

Positive staining for $\alpha_{1}$ antitrypsin was seen seven $(70 \%)$ of control specimens and in 34 $(65 \%)$ of the pancreatitis specimens. Comparison of hepatic sinusoidal macrophage numbers in periportal and perivenous zones of both control and pancreatitis liver specimens showed no significant difference in distribution. In addition, there was no significant difference between the incidence of macrophages in the control and pancreatitis groups. In both control and pancreatitis liver specimens, these mononuclear cells exhibited a more diffuse distribution than lymphocytes, being seen throughout the liver parenchyma as well as in portal regions (Figs 3 and 4). The density of macrophages, as defined by staining for lysozyme, was mean (SEM) 23 (5) cells per high power field (hpf) for controls and 20 (2) cells per hpf for pancreatitis specimens. Using $\alpha_{1}$ antitrypsin as the criterion for identifying macrophages, these cells numbered 10 (5) per hpf for controls and 20 (3) per hpf for pancreatic specimens.

\section{COMPARISON BETWEEN ALCOHOLICS AND NON-} ALCOHOLICS

Data comparing histopathological features in patients with an alcoholic aetiology for their

TABLE III Density of lymphoid infiltrates in portal tracts of pancreatitis biopsy specimens $(n=52)$

\begin{tabular}{lll}
\hline & \multicolumn{2}{l}{ No of positive specimens } \\
\cline { 2 - 3 } Mean no of cells per portal tract & $T$ cells & B cells \\
\hline $1-25$ & 17 & 8 \\
$26-50$ & 14 & - \\
$51-75$ & 9 & - \\
$76-100$ & 4 & - \\
$>100$ & 3 & - \\
\hline
\end{tabular}

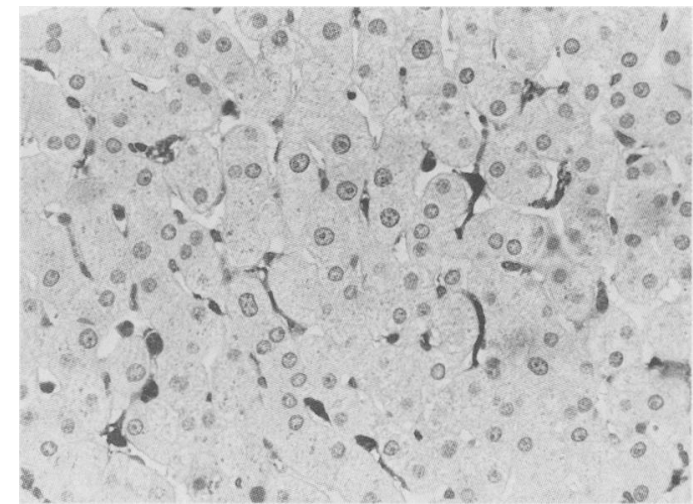

Figure 3: Liver biopsy specimen stained using polyclonal antiserum to lysozyme. The identified macrophages and histiocytes were predominantly lining sinusoids of parenchymal zone I. A few morphologically identical sinusoidal lining cells remained unstained (original magnification $\times 480$ ).

pancreatitis and those with other causes are presented in Table IV. Statistical analysis, where possible, has shown no differences between the two groups except that $\mathrm{T}$ lymphocytes were more frequent in alcoholic patients $(\mathrm{p}<0 \cdot 05)$.

\section{Discussion}

The incidence of clinically manifest liver disease, such as cirrhosis and portal hypertension, is not well defined in patients with alcoholic chronic pancreatitis; it is estimated to be about $10 \% .^{3-5}$ Recent histopathological studies have identified cirrhosis in $2-12 \%$ of patients with chronic pancreatitis, although abnormal liver histology was reported in up to $60-70 \%$ of these cases. ${ }^{6} 11$ Most of these changes have been described as non-specific and mild.

In this series, none of the 52 liver biopsy specimens in patients with pancreatitis showed evidence of serious liver disease. Features of alcohol associated liver disease, including parenchymal liver cell damage, Mallory hyaline bodies, and pericellular fibrosis within the parenchyma of hepatic biopsy specimens were not seen. ${ }^{12}$ Although perivenular fibrosis has been suggested as a marker of future cirrhosis, this finding was inconsistent and not associated with other characteristics of alcoholic liver damage. ${ }^{1314}$ In none of our patients was there any appreciable

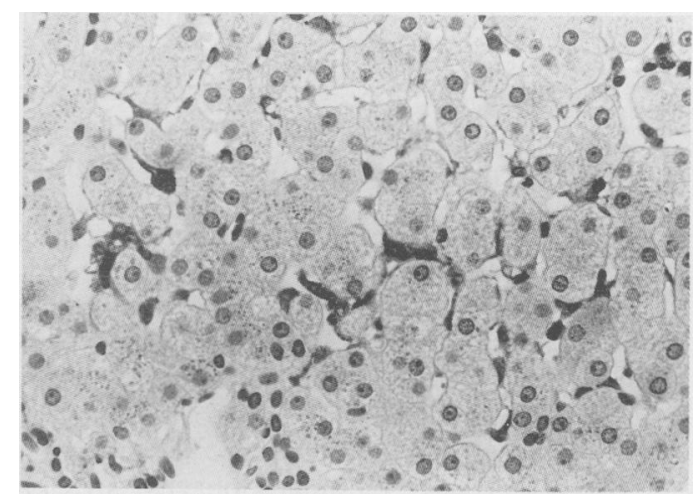

Figure 4: Polyclonal antiserum to $\alpha_{l}$ antitrypsin identifying cells of myeloid/macrophage type lining parenchymal sinusoids. In common with the distribution of similar cells identified using antilysozyme, very few such cells were present within portal tracts (original magnification $\times 480$ ). 
TABLE IV Histological features of alcoholic and non alcoholic patients with chronic pancreatitis

\begin{tabular}{llrlrl}
\hline \multicolumn{5}{c}{$\begin{array}{l}\text { Alcoholic } \\
(n=40)\end{array}$} & \multicolumn{3}{l}{ Non-alcoholic } \\
& No $n$ 12) & \\
& No & $(\%)$ & No & $(\%)$ & p value \\
\hline Portal fibrosis & 0 & $(0)$ & 0 & $(0)$ & - \\
Steatosis & 12 & $(30)$ & 6 & $(50)$ & $0.5>\mathrm{p}>0.1$ \\
Cholestasis & 3 & $(8)$ & 2 & $(17)$ & $0.5>\mathrm{p}>0.1$ \\
Iron & 10 & $(25)$ & 1 & $(8)$ & $0.5>\mathrm{p}>0.1$ \\
Copper & 2 & $(5)$ & 0 & $(0)$ & - \\
UCHL1 (T cell) & 38 & $(95)$ & 9 & $(75)$ & $0.05>\mathrm{p}>0.02$ \\
L26 (B cell) & 8 & $(20)$ & 0 & $(0)$ & - \\
Lysozyme & 34 & $(85)$ & 9 & $(75)$ & $0.5>\mathrm{p}>0.1$ \\
$\alpha_{1}$ Antitrypsin & 25 & $(63)$ & 9 & $(75)$ & $0.5>\mathrm{p}>0.1$ \\
\hline
\end{tabular}

increase in fibrous connective tissue within portal tracts nor was there evidence of cirrhosis. The remarkable absence of severe liver damage, especially in the group of heavy drinkers, stands in sharp contrast to the severe changes of chronic pancreatitis observed in nearly every case.

The commonest hepatic abnormality was steatosis, which was present in $35 \%$ of patients but severe in only $13 \%$. In only one patient was this associated with abnormal liver function. Hepatocellular fat is labile and may therefore reflect the patient's alcohol intake shortly before operation rather than any significant liver cell dysfunction. ${ }^{12}$

The $10 \%$ incidence of cholestasis is in keeping with the recent Glasgow experience. ${ }^{6}$ Cholestasis probably reflects extrahepatic biliary obstruction, which was radiologically confirmed in al five of our patients. ${ }^{7-9}$ Cholestasis was not seen in five other patients with biochemical, or radiological, or both, evidence of bile duct stenosis. Marginal ductular proliferation, another indicator of recent bile duct stenosis, was present in only one of the five specimens showing cholestasis but was identified in an additional three patients. Intrahepatocyte copper, a feature suggesting a chronic cholestatic disorder in the absence of cirrhosis, ${ }^{15}$ was not observed in any of our cases with cholestasis.

In this present series, we have defined the characteristic features of inflammatory infiltration of the liver in chronic pancreatitis. Lymphoid infiltration, seen predominantly in portal areas, comprised $\mathrm{T}$ lymphocytes rather than B lymphocytes. Only four patients had a detectable parenchymal distribution of $T$ lymphocytes. Since all four specimens contained a very dense portal infiltration, these parenchymal lymphocytes probably reflect a 'spill over' mechanism. In the absence of features associated with hepatocyte toxicity, our data suggest that the presence of $T$ lymphocytes within the parenchyma and portal tracts probably reflects an upward migration of inflammatory cells from the pancreas rather than a direct hepatotoxic process.

The presence and density of macrophages within liver biopsy specimens from patients with suspected alcohol associated chronic pancreatitis is also of note. Several studies have shown cells of the monocyte macrophage system to be phenotypically heterogeneous. ${ }^{16}$ Human hepatic macrophages are known to originate from circulating monocytes of bone marrow origin. ${ }^{18}$ They form a self replicating population of resident macrophages which remains stable unless a major inflammatory stimulus affects the liver. ${ }^{19}$ Immunohistological examination has indicated the numbers of lysozyme positive hepatic sinusoidal macrophages to be decreased in alcohol associated liver disease.$^{20}$ During that study, an undefined portion of sinusoidal lining cells was found to be negative for lysozyme. A subsequent study confirmed these findings and showed portal tract macrophages to be increased in liver biopsy specimens exhibiting only steatosis whereas lysozyme positive hepatic sinusoidal macrophages were decreased. ${ }^{21}$ Heterogeneity of liver macrophage phenotypes was confirmed by the disparity of numbers of macrophages identified using three different immunohistochemical markers.

In this study, the numbers of macrophages in control liver biopsy specimens and in those from patients with chronic pancreatitis were not significantly different. These observations are distinct from the findings in alcoholic liver disease $^{2021}$ and hence provide additional evidence that alcoholic liver disease and alcohol associated chronic pancreatitis do not involve exactly similar pathogenetic mechansims. Differences in numbers of macrophages identified using antisera to lysozyme and to $\alpha_{1}$ antitrypsin further substantiate the phenotypic heterogeneity of hepatic macrophages in both control and pancreatitis biopsy specimens. Differences in aetiology and subsequent pathogenesis of chronic pancreatitis, thus differentially modulating the phenotype of hepatic macrophages, may account for the absence of lysozymal staining in nine of the specimens from patients with pancreatitis.

It is not understood why patients with alcoholic damage to the pancreas so seldom have serious liver disease. Although nutritional factors have been implicated, ${ }^{22} 23$ recent reports do not support this hypothesis. ${ }^{24-26}$ The original suggestion that a diet rich in fat and protein might predispose to alcoholic liver injury of the pancreas was contradicted by the subsequent dietary studies. Also, alcohol consumption is notoriously difficult to quantify, particularly in relation to nutritional status. If chronic pancreatitis occurs earlier than alcoholic liver disease, affected patients might not have time to develop hepatic cirrhosis. ${ }^{3}$ Similarly, ethanol intake might change with the onset of pancreatic pain or the threat of operation. Yet evidence of residual hepatic disease secondary to alcohol would be expected, but this was not found. Since certain human leukocyte antigen (HLA) haplotypes are associated with either alcoholic liver disease or chronic pancreatitis, genetic factors could help to determine which disease process develops; however, current data are inconclusive and require further elaboration. ${ }^{27-29}$ Lastly, it is known that in chronic pancreatitis liver and pancreatic damage proceed independently. ${ }^{30}$

Current evidence indicates that chronic pancreatitis is not a single defined entity, but a disease process comprising a group of different aetiologies and pathogenetic mechanisms which share a few common morphological end points. If a single agent, such as alcohol, is the most important aetiological factor in the genesis of this disease, then the exocrine pancreatic tissues of affected patients, when compared with hepatic 
parenchymal tissue, seem to be unduly sensitive to its toxic effects. Such sensitivity is either congenital or acquired in origin. The finding of raised numbers of $T$ lymphocytes infiltrating hepatic portal tracts, particularly in the absence of acute inflammatory cells, might indicate that a cell mediated immune component is involved in human chronic pancreatitis. We suggest that ethanol is not acting as a simple toxin in a dose related manner but rather as an adjunct or 'trigger' that renders exocrine pancreatic tissue sensitive to cell mediated cytotoxicity.

We are grateful for the assistance of Professor J W B Bradfield, Department of Histopathology, University of Bristol in permitting unreserved access to the archived specimens at the Royal Infirmary.

1 Read G, Braganza JM, Howat HT. Pancreatitis - a retrospective study. Gut 1976; 17: 945-52.

2 MacLaren IF. Observations and surgical management of chronic pancreatitis in the British Isles. A review of the twentieth century. World F Surg 1990; 14: 19-27.

3 Angelini G, Merigo F, Degani G, et al. Association of chronic alcoholic liver and pancreatic disease: a prospective study. Am $\mathcal{F}$ Gastroenterol 1985; 80: 998-1003.

4 Sarles H, Sarles JC, Camette R, et al. Observations in 205 confirmed cases of acute pancreatitis, recurring pancreatitis and chronic pancreatitis. Gut 1965; 6: 545-52.

5 Strum WB, Spiro HM. Chronic pancreatitis. Ann Intern Med 1971; 74: 264-77.

6 Wilson C, Auld CD, Schlinkert R, et al. Hepatobiliary complications in chronic pancreatitis. Gut 1989; 30: 570-7.

7 Afroudakis A, Kaplowitz N. Liver histopathology in common bile duct stenosis due to chronic alcoholic pancreatitis. Hepatology 1981; 1: 65-72.

8 Aranha GV, Prinz RA, Freaark RJ, Greenlee HB. The spectrum of biliary tract obstruction from chronic pancreatitis. Arch Surg 1984; 119: 595-600.

9 Scott J, Summerfeld JA, Elias E, Dick R, Sherlock S. Chronic pancreatitis: a cause of cholestasis. Gut 1977; 18: 196-201.

10 Braillon A, Capron JP, Hervé MA, et al. Liver in obesity. Gut 1985; 26: 133-9.

11 Dutta SK, Mobrahan S, Iber FL. Associated liver disease in chronic pancreatitis. Dig Dis $1978 ; 23: 618-22$

12 Scheuer PJ. Liver disease in the alcoholic. In: Scheuer PJ.
Liver biopsy interpretation. London: Baillière Tindall, 1988: 83-98.

13 Nokano M, Worner TM, Lieber CS. Perivenular fibrosis in alcoholic liver injury: ultrastructure and histologic progression. Gastroenterology 1982; 83: 777-85.

14 Worner TM, Lieber CS. Perivenular fibrosis as precursor lesion of cirrhosis. FAMA 1985; 254: 627-30.

15 Guarascio P, Yentis F, Cevikbas U, Portmann B, Williams R. Value of copper-associated protein in diagnostic assessment of liver biopsy. 7 Clin Pathol 1983; 36: 18-23.

16 Cline MJ, Lehrer RI, Territo MC, Golde DW. Monocytes and macrophages: functions and diseases. Ann Intern Med 1978; 88: 78-88

17 Radzun HJ, Parwaresch MR. Differential immunohistochemical resolution of the human mononuclear phagocyte system. Cell Immunol 1983; 82: 174-83.

18 Gale RP, Sparkes RS, Golde DW. Bone marrow origin of hepatic macrophages (Kupffer cells) in humans. Science 1978; 201: 937-8

19 Summerfield JA, Jones EA. Further progress towards understanding hepatic sinusoidal cells. $\mathcal{H}$ Hepatol 1986; 3:413-8.

20 Mills LR, Scheuer PJ. Hepatic sinusoidal macrophages in alcoholic liver disease. F Pathol 1985; 147: 127-32.

21 Karakucuk I, Dilly SA, Maxwell JD. Portal tract macrophages are increased in alcoholic liver disease. Histopathology 1989; 14: $245-53$

22 Sarles H. An international survey of nutrition and pancreatitis. Digestion 1973; 9: 389-403.

23 Uscanga L, Robles-Diaz G, Sarles H. Nutritional data and aetiology of chronic pancreatitis in Mexico. Dig Dis $\mathrm{Sc}$ 1985; 30: 110-3.

24 Pitchumoni CS, Sonnenschein M, Candido FM, Panchacharam P, Cooperman JM. Nutrition in the pathogenesis of alcoholic pancreatitis. Am $\mathcal{F}$ Clin Nutr 1980; 33: 631-6.

25 Wilson JS, Bernstein L, MacDonald C, Tait A, McNeil D, Pirolla RC. Diet and drinking habits in relation to the Pirolla RC. Diet and drinking habits in relation to the
development of alcoholic pancreatitis. Gut 1985;26: 882-7.

26 Mezey E, Kolman CJ, Diehl AM, Mitchell MC, Herlong HF. Alcohol and dietary intake in the development of chronic pancreatitis and liver disease in alcoholism. Am $\mathcal{F}$ Clin Nutr 1988; 48: 148-51.

27 Eddleston ALWF, Davies M. Histocompatibility antigens in alcoholic liver disease. Br Med Bull 1982; 38: 13-6.

28 Saunders JB, Wodak AD, Williams R. What determines susceptibility to liver damage from alcohol? $\mathcal{F} R$ Soc Med 1984; 77: 204-16.

29 Forbes A, Schwarz G, Mirakian R, et al. HLA antigens in chronic pancreatitis. Tissue Antigens 1987; 30: 176-83.

30 Sandilands D, Jeffrey IJM, Haboubi NY, MacLennan IAM, Braganza JM. Abnormal drug metabolism in chronic pancreatitis - treatment with antioxidants. Gastroenterology
1990; 98: 766-72. 